Revue critique de fixxion française contemporaine

22 | 2021

Figures du mensonge et de la mauvaise foi dans le roman contemporain

Le "je" en porte-à-faux. Pratiques déceptives de l'écriture de soi chez Marie NDiaye et Camille Laurens

Anne-Sophie Donnarieix

(2) OpenEdition

Édition électronique

URL : https://journals.openedition.org/fixxion/344

DOI : 10.4000/fixxion.344

ISSN : 2295-9106

Éditeur

Ghent University

Référence électronique

Anne-Sophie Donnarieix, "Le "je" en porte-à-faux. Pratiques déceptives de l'écriture de soi chez Marie NDiaye et Camille Laurens ", Revue critique de fixxion française contemporaine [En ligne], 22 | 2021, mis en ligne le 15 juin 2021, consulté le 17 février 2022. URL : http://journals.openedition.org/fixxion/344 DOI : https://doi.org/10.4000/fixxion.344

Ce document a été généré automatiquement le 17 février 2022.

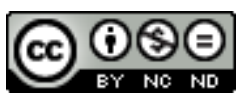

Les contenus de la Revue critique de fixxion française contemporaine sont mis à disposition selon les termes de la licence Creative Commons Attribution - Pas d'Utilisation Commerciale - Pas de Modification 4.0 International. 


\title{
Le "je" en porte-à-faux. Pratiques déceptives de l'écriture de soi chez Marie NDiaye et Camille Laurens
}

\author{
Anne-Sophie Donnarieix
}

1 L'autobiographie ne compte généralement pas parmi ces genres littéraires dans lesquels le narrateur a pour habitude de mentir, et l'idée de duplicité narrative semble de prime abord mal s'accommoder d'un pacte de lecture qui présuppose une exigence de sincérité ${ }^{1}$. On sait certes que la forme autobiographique a fait les frais de la modernité : des autobiographies fictives de Nabokov à l'autofiction de Doubrovsky, des stratégies d'empêchement chez Sarraute à la "nouvelle autobiographie" de RobbeGrillet, elle s'est émancipée d'un certain modèle structurel où l'avaient circonscrite des traditions séculaires, quitte à troquer la vérité contre l'invention fictionnelle, et l'écriture de soi contre celle de son impossibilité. Mais la fiction n'équivaut pas au mensonge, et si ces dissidences autobiographiques renouvellent les modes de saisie du sujet, elles ne mettent toutefois pas nécessairement en cause la bonne volonté du narrateur.

2 C'est bien pourtant la notion de dissimulation qui caractérise le mensonge dans son acception la plus courante. La définition n'est pas nouvelle et remonte au moins au De mendacio d'Augustin : le menteur n'est pas celui qui dit le faux, mais celui qui "désire tromper" en énonçant ${ }^{2}$. Précisons toutefois que si elle forme une partie intégrante de son fonctionnement structurel, la "tromperie" ne constitue pas nécessairement la finalité de tout mensonge, contrairement aux conclusions d'Augustin qui fonde à partir de là sa condamnation morale absolue du mensonge ${ }^{3}$. Il s'avère du reste difficile de taxer un roman de quelque volonté de nuisance à l'égard de son lectorat - fût-ce Le meurtre de Roger Ackroyd. Débarrassée de ses présupposés éthiques, l'idée de tromperie ou de dissimulation offre pourtant un éclairage précieux sur la question du mensonge. Jochen Mecke, dans la définition tripartite qu'il en propose, insiste d'ailleurs précisément sur ce point : le mensonge résulterait d'une divergence entre sentiment et expression (1), divergence dissimulée (2) et mise au service d'une finalité qui reste 
également tue (3) ${ }^{4}$. De ce point de vue, on peut envisager que le mensonge n'engage pas tant le contenu du discours (vrai vs. faux, fiction vs. non-fiction) qu'une particularité de l'ethos discursif fondé sur une pratique déceptive, liée à la duplicité, à la dissimulation, à la tromperie, à la mystification. On pense à certains types de narrations "non fiables" 5 qui mettent en place un système de transmission défaillant où l'instance narrative prend un malin plaisir à duper lectrices et lecteurs, mais aussi à quelques cas d'impostures littéraires ${ }^{6}$ (du Pierre Ménard borgésien aux écrivains fictifs de JeanBenoît Puech ou d'Antoine Volodine). Ces différents mécanismes de tromperie impliquent une transgression plus insidieuse qui excède la seule opposition du vrai et du faux : ils détournent ou obstruent le système communicationnel et instiguent un doute face à des instances narratives ou auctoriales visiblement réticentes à livrer leurs secrets.

3 Ces derniers restent toutefois problématiques dans le cas de l'écriture de soi - qui nous intéresse ici -, puisque ce même ethos discursif (qu'il engage ou non la fiction) se fonde sur un postulat d'authenticité. On connaît la formule emphatique d'un Jean-Jacques jurant devant le tribunal de ses pairs et devant les trompettes du Jugement dernier de ne se peindre qu'"exactement d'après nature et dans toute sa vérité"7. L'autofiction elle-même n'y déroge guère si l'on accepte de la définir avec Chloé Delaume comme un art de "mentir au plus juste", en excédant au besoin le carcan trop étroit de la nonfiction pour donner à voir une réalité subjective, moins exacte certes, mais sans doute aussi sincère. Qu'en est-il en revanche lorsque l'instance narrative prend un plaisir sensible à mener son lectorat en bateau, et que la manipulation devient le parangon d'une esthétique autobiographique fondée sur la dissimulation ou sur le brouillage des pistes de lecture référentielles? Je tenterai de répondre à cette question en détaillant les pratiques déceptives à l'œuvre dans Autoportrait en vert de Marie NDiaye (2005) et Celle que vous croyez de Camille Laurens (2016) ${ }^{9}$. Il s'agira d'abord d'étudier ces textes depuis une perspective narratologique, puis d'interroger leur portée subversive dès lors que ces procédés impliquent aussi une rupture normative - envers les normes littéraires du récit de soi auxquelles les autrices se plaisent à déroger, mais également envers des normes socioculturelles plus insidieuses dont les textes exposent le caractère fallacieux.

\section{Effacer les traces : Autoportrait en vert}

4 Autoportrait en vert constitue une pièce singulière dans l'œuvre narrative de Marie NDiaye. Plus résolument intimiste que la plupart des romans de l'autrice, plus bref aussi dans sa facture, le récit est en outre rédigé dans une langue surprenante de sobriété syntaxique - loin de l'écriture proustienne aux phrases déliées, d'une complexité langoureuse et obsédante, qui caractérise le plus souvent sa plume. Autre particularité, suffisamment rare pour être notable : le titre semble annoncer de prime abord un récit à dimension autobiographique. Quelques indices laissés ici et là pourraient presque étayer l'hypothèse : la narration se fait à la première personne du singulier, mentionne un mari nommé Jean-Yves, une maison en province bordelaise, un père africain, des activités littéraires. Le récit empêche néanmoins - bien sûr - une coïncidence trop parfaite: le nombre d'enfants ne correspond pas, les prénoms non plus, et le voile fantastique qui plane sur le récit ne laisse que peu de doute quant à l'authenticité des faits ${ }^{10}$. Pour autant, le récit, à cet égard, ne "ment" pas : il prend 
d'ailleurs soin de ne pas se présenter comme une autobiographie et le titre "autoportrait en vert", convoque d'emblée une double entrave générique (par le vecteur pictural d'une part, de l'autre par le filtre "vert" qui révèle tout au long du texte sa puissance de déréalisation ${ }^{11}$ ). Nul "pacte" n'est donc conclu, ni romanesque ni autobiographique, ou plutôt si : mais le pacte en question s'engage précisément à se soustraire à l'un comme à l'autre. Le livre en somme remplirait ses promesses en nous livrant un portrait déformé, inauthentique de l'autrice.

5 La situation se complique toutefois en ce que la narration met en place un système d'obstruction généralisé qui vise à empêcher toute transparence du discours, quitte à flirter régulièrement avec la sincérité ou à verser plus délibérément dans la mystification. D'une part, la narration prend soin d'éviter toute écriture thématique de soi : la narratrice se refuse à "parler d'elle" et néglige son rôle de protagoniste au point de substituer au récit de sa vie le récit de la vie des autres, ces femmes "en vert" qu'elle décrit, épie ou invente. L'ouverture du livre est à cet égard emblématique : la voix narrative (anonyme) se tapit dans l'ombre d'une Garonne verte et obsédante qui engloutit l'espace du récit sous la description anxiogène de ses crues ("C'est le soir et le niveau de la Garonne monte heure après heure dans l'obscurité" (AV 9)). Celle-ci est relayée plus tard par différents portraits féminins qui accapareront à leur tour le récit, ne dévoilant de la narratrice que son regard tendu, crispé vers ces figures énigmatiques qui fonctionnent comme autant de barrages habilement disposés pour empêcher tout épanchement introspectif. Le récit de soi est dissimulé, il n'advient qu'à rebours, en négatif, comme soumis à un principe elliptique du reste assumé par la narratrice : "quelque chose d'impalpable, un voile, une lueur d'irréalité me rendent réticente à [...] livrer qui je suis" (AV 32). Et alors même que la focalisation interne devrait logiquement privilégier l'accès à la vie intérieure de cette dernière, c'est ici surtout son opacité qu'elle illustre, dans une lignée qui s'inscrirait à mi-chemin entre les protagonistes de Kafka et L'étranger de Camus ${ }^{12}$. Du “je", nous n'apprenons que des bribes, et l'attention de la narratrice se reporte inlassablement sur les autres - environnement direct ou personnages également évanescents, inaccessibles, rétifs, qui se refusent à son regard et mettent ainsi en abyme une certaine impossibilité heuristique: celle de la connaissance des autres comme de la connaissance de soi $^{13}$.

6 Cette double dissimulation du "je" est soutenue par un principe de brouillage onomastique et pronominal. L'emploi des pronoms personnels joue en effet un rôle ambigu dans le récit : ceux-ci désignent en même temps qu'ils dissimulent, et simulent un savoir commun au locuteur et à l'allocutaire qui fait pourtant souvent défaut. C'est ainsi un "nous" indéterminé, flottant, qui ouvre le récit, créant une communauté fictive et vaguement hostile à laquelle succèdera un "je" tout aussi anonyme et inassignable. Jouant d'une fonction déictique qui ne renvoie à aucun signifié préalablement défini ${ }^{14}$, ces pronoms "nous" et "je" confrontent le lecteur à une indétermination linguistique : de qui parle-t-on? Ce mécanisme grammatical, qui suppose l'inclusion du lectorat, repose ici au contraire sur une exclusion latente de ce dernier, privé d'un savoir que le texte se plaît à lui refuser tout en démultipliant les occurrences de pronoms inassignables. Loin de lever ces incertitudes, l'arrivée des premiers noms propres contribue à embrouiller davantage toute entreprise référentielle :

C'est alors que je tombe sur Cristina, mais dès l'instant où je la vois je ne sais plus si c'est elle, ou si c'est Marie-Gabrielle, ou si c'est Alison - non pas que m'échappe son prénom exact: entre ces trois femmes, je ne sais simplement plus qui est celle-ci.

[...] Celle qui est peut-être Cristina est une jeune femme, aussi elle est en short, 
élastique et moulant, imprimé de fleurs vertes sur fond vert. Un peu de ma jubilation se dérobe. Je pense à me méfier [...]. Si cette femme est bien Cristina, je me rappelle qu'elle est mon amie. Cristina a plus de droits à ce titre que MarieGabrielle ou Alison qui ne sont, pour autant que je m'en souvienne, que de bonnes et franches camarades à qui on ne songerait pas à se confier. [...] Ai-je jamais révélé quoi que ce soit à Cristina ? Certainement pas, c'est contraire à ma nature. ( $A V$ 21-22)

7 Sous couvert de présenter le personnage de Cristina ( ?) et de lui donner une certaine solidité grammaticale, l'accumulation de présentatifs et de démonstratifs ("c'est", "c'est elle", "celle qui", “celle-ci") rend confus les propos de la narratrice à son sujet, et contribue à créer de multiples identités autonomes et discordantes qui viennent renforcer (ou traduire) l'incertitude onomastique ${ }^{15}$. Ce malaise dans la coïncidence des personnages avec eux-mêmes fonctionne bien sûr comme le reflet d'une narration autodiégétique elle-même retorse. En témoigne un discours ambigu, dont les incises répétées ("pour autant que je m'en souvienne", "si je me souviens bien"), le mode interrogatif obsédant (“Ai-je jamais révélé quoi que ce soit [...] ?”) finissent par éveiller une certaine suspicion à l'égard de la fiabilité de la voix narrative.

Celle-ci est en effet mise à mal par les multiples transgressions d'un régime autodiégétique qui implique un champ de vision relativement réduit. Vers le milieu du récit pourtant, la narration feint une curieuse omniscience, et entreprend de narrer l'histoire d'un personnage secondaire, Jenny (une amie de la protagoniste), depuis la position surplombante et improbable d'un narrateur capable d'ausculter avec une acuité déroutante le for intérieur de ses personnages, ce qui pose évidemment problème du point de vue de la logique narrative :

11 mars 2001 - Jenny s'en revient chez ses parents, à la campagne, car elle n'a plus de quoi payer son loyer. Ils l'accueillent dans un silence douloureux et gêné. Ils ne peuvent tout à fait croire qu'elle ait échoué à ce point, elle à qui ils rendaient visite avec une timidité due à leur crainte de ne pas être à la hauteur de sa prospérité. Elle comprend qu'ils se demandent, désemparés mais sévères : lui reste-t-il, à notre fille, un peu de dignité ? (AV 48-49)

9 Nous retrouvons là la définition canonique du narrateur non fiable selon Wayne C. Booth $^{16}$ : la fiabilité du narrateur ne tient pas à la véracité de son discours (invérifiable en l'occurrence), mais avant tout à l'écart que ce discours instaure avec les codes tacitement mis en place par le texte (narration autodiégétique, restriction du champ de connaissance). À la rétention d'informations sur la protagoniste succède ici - mais c'est le revers d'une même médaille - une prolifération de détails sur les autres que rien ne légitime, et décrits depuis une position narrative incohérente et plurifocalisée :

30 mars 2001 - [Jenny] me raconte longuement au téléphone que son histoire d'amour avec Ivan a duré plusieurs années avant qu'elle ne décide de quitter la province, et malgré l'éloignement j'imagine son visage durci et sa mâchoire contractée car j'entends une voix cassante et froide qui n'est pas la voix de Jenny habituellement et qu'elle se force à prendre, me semble-t-il, pour éloigner la sentimentalité. Jenny n'est pas une femme sentimentale. N'est-ce pas témoigner d'une pathétique complaisance, pense Jenny, que de se laisser porter par un romantisme qu'on n'avait pas dans sa jeunesse, simplement parce qu'on est désœuvré [...] ? Certainement, pense Jenny, le romantisme après coup est pitoyable, dérisoire, médiocre. Comment faire cependant ${ }^{17}$ (AV 53-54)

Le changement subtil de focalisation qui passe de la protagoniste au personnage de Jenny (et $\mathrm{du}$ discours indirect au discours direct) déstabilise d'autant plus qu'il se présente lui-même comme trompeur : la répétition exacte du syntagme "pense Jenny" 
à deux lignes d'écart semble avertir de l'artificialité du procédé tandis que l'explication de la supercherie - "elle ne dit rien de tout cela. Je le devine" (AV 54) - arrive trop tardivement pour réhabiliter la bonne foi de la narratrice.

Dans un article consacré aux narrations non fiables, Francis Langevin distingue deux types de narrateur: le narrateur "faillible", trompé par ses propres perceptions (narrations enfantines, ignorance, handicap cognitif etc.), et le narrateur véritablement "trompeur" qui cherche à manipuler son lecteur ${ }^{18}$. Dans le cas d'Autoportrait en vert, la distinction se révèle délicate, et la frontière entre les deux est rendue poreuse par une instance narrative certes habile à brouiller les pistes, mais dont la capacité à appréhender le monde de manière rationnelle est aussi remise en cause - en témoignent ces spectres évanescents (imaginaires?) qui se dressent régulièrement sur son parcours. En ce sens, on pourrait attribuer, en partie du moins, ces étrangetés narratives au mode de perception fantastique et irrationnel d'une conscience largement énigmatique, et les mettre sur le compte d'un "exotisme" ${ }^{19}$ cognitif. Il n'en reste pas moins que cette subjectivisation radicale du récit n'est pas exempte de manipulation : ainsi du brouillage minutieux des repères temporels censés structurer le récit, et qui, disposés pêle-mêle $(2003,2002,2003,2001,2003)$ perturbent au contraire sa linéarité. Une autre incohérence de dates, infime mais révélatrice, mérite d'être mentionnée: en 2003, lit-on, la narratrice est âgée d'une quarantaine d'années ("Comme il est curieux, après qu'on a côtoyé sa propre mère pendant une quarantaine d'années" (AV 72)) - et l'on apprend pourtant une page plus loin que cette même mère, en l'an 2000, "avait quarante-cinq ans" (AV 73). Erreur? Oubli? Rajeunissement fantastique de la mère ${ }^{20}$ ? Duperie facétieuse adressée en pied de nez à une lectrice trop tatillonne? Le doute plane, mais ces décalages temporels assoient le caractère mystificateur d'une instance narrative qui ne cesse de se dérober et multiplie les embûches comme pour mieux semer ses poursuivants.

Dispersion des traces et surexposition des mécanismes de brouillage : l'écriture de soi revêt chez Marie NDiaye les attraits paradoxaux d'un "je" rétif, qui ne se livre que par dissimulation. Tout de feintise ténue mais entêtante, celui-ci se déploie dans les interstices laissés par l'horizon d'attente autobiographique que ne manque pas de susciter le titre, et se plaît justement à effacer les traces référentielles, à se cacher derrière une narration ambiguë et à pratiquer la rétention d'informations (référents grammaticaux absents, flottement trompeur de l'ancrage temporel). Le récit reste toutefois à cet égard moins mensonger que déceptif: il ne s'agit pas de tromper en "énonçant le faux" mais plutôt - paradoxalement - de ne rien dire, de dévoiler le moins possible et d'étouffer chaque indice sous une chape d'ambiguïté, dans le sillage direct du principe de double dissimulation (structurelle et pragmatique) mis en lumière par Jochen Mecke.

\section{Démultiplications métaleptiques : Celle que vous croyez}

13 Dans le roman Celle que vous croyez de Camille Laurens, paru en 2016, le caractère déceptif $d u$ récit autobiographique tire sa force d'un procédé inverse, qui ne consiste plus à effacer les traces mais au contraire à les démultiplier, étouffant sous ses proliférations d'avatars fictionnels les pistes référentielles esquissées par le texte. Unies par un même motif - celui du désir et de la manipulation séductrice -, sept histoires se succèdent, s'imbriquent les unes dans les autres, se prolongent, s'infirment 
ou se réécrivent de manière à rendre indécidable tout texte "premier" ou originaire, et à brouiller à l'envi les différentes échelles narratives par un chassé-croisé de métalepses qui font de l'autrice le personnage de sa propre fiction (ou inversement ${ }^{21}$ ). Seule la trame reste plus ou moins semblable : une femme décide de créer un faux profil de jeune fille sur les réseaux sociaux pour épier son mari volage, et finit par séduire l'un de ses amis qui tombera à son tour dans les rets d'une duperie virtuelle aux conséquences variables et le plus souvent funestes.

S'il est aisé de rattacher le roman au genre de l'autofiction ${ }^{22}$, les indices se font pourtant attendre, retardés notamment par les enchevêtrements de récits que rien ne rattache, dans un premier temps, à la figure auctoriale. Toute la première moitié du roman porte ainsi sur quatre portraits d'une (même ?) femme, jeune enseignante de lettres divorcée, d'abord mise en scène dans un commissariat de police, puis dans un centre psychiatrique où elle apparaît sous le nom de Claire, puis enfin dans le compterendu de son psychiatre qui présente par le biais d'un récit enchâssé un texte autofictionnel écrit par "Claire" dans le cadre d'un atelier thérapeutique mené par une certaine "Camille". Ce n'est que dans la deuxième moitié du roman que "Camille" (la même ?) prend la parole à la première personne, déguisée sous les traits de l'autrice (Camille Laurens) pour écrire à son éditeur et lui "raconter la vraie histoire, l'histoire vraie, celle qui [lui] est arrivée" (CVC 139) - celle, déformée, de Claire/Camille. De Claire(s) en Camille(s) (et avec l'aide de quelques Katia(s)), tout un réseau d'avatars féminins se dresse en simulacres les uns des autres. Ces derniers prennent alternativement le rôle d'un "moi" tissé en toile arachnéenne, composé d'une petite dizaine de personnages et de narratrices oscillant entre le "je" et le "elle". Si l'alternance des pronoms n'est pas en soi une transgression autobiographique Philippe Lejeune rappelle qu'“il peut parfaitement y avoir identité du narrateur et du personnage principal sans que la première personne soit utilisée" ${ }^{23}$ - elle se donne à lire ici dans une perspective résolument déceptive qui cherche à fausser les pistes et met en place une identité polycéphale parfaitement indécryptable.

D'autres procédés narratologiques font en outre basculer le roman vers des pratiques plus nettement mystificatrices. Les multiples instances narratives (1/une femme, 2/ Claire-patiente, 3/le psychiatre, 4/Claire-personnage, 5/Camille-autrice, 6/Camillepersonnage $7 /$ le mari de Claire) ont en effet la particularité de se trouver dans une même situation d'énonciation - une situation délicate : elles sont toutes sommées de se justifier. À l'une, on reproche son imposture virtuelle, à l'autre, un possible plagiatpsychique $^{24}$, au psychiatre, une faute professionnelle, au mari enfin, de douteuses manigances conjugales. Les discours sont ainsi faussés d'emblée dans leur postulat de sincérité : la parole délivrée (le récit lu) est contrainte, partiale, intéressée, et soumise à un dispositif énonciatif qui rappelle à certains égards celui de l'interrogatoire ${ }^{25}$ en ce qu'il se fonde sur la présence implicite d'un destinataire-juge qu'il importe de persuader de son innocence, quitte à enjoliver les faits. "Déposition $\mathrm{n}^{\circ} 453 \mathrm{JA}$ ", "entretiens avec le docteur Marc B.", "audition du docteur Marc B.", "Cabinet de Me Deligne, avocat" - les paratextes soulignent déjà ces rapports de pouvoir qui motivent le récit en liant gendarmes et suspects, patients et psychiatres, écrivains et avocats, maris adultères et juges d'instruction. Et de fait: chaque personnage-narrateur manipule sans vergogne son interlocuteur, usant régulièrement de flatteries hypocrites ou de pièges destinés à tester la sagacité de l'allocutaire :

J'ai déjà tout raconté dix fois à vos collègues, vous n'avez qu'à lire mon dossier. [...]

C'est votre premier poste? Car vous n'avez que trente ans tout au plus. 
Vous ne les faites pas.

Je ris parce que je vous récite du Marivaux et que vous n'y voyez que du feu. [...]

Vous pourriez le sentir, je ne sais pas, au rythme, à l'intonation. C'est votre métier

d'entendre comment ça sonne. De repérer ce qui cloche. (CVC 17)

La filiation intertextuelle et la référence théâtrale aux Fausses confidences (dont le titre est en effet programmatique ${ }^{26}$ ) se révèlent éclairantes : chaque fragment d'histoire peut en effet se lire comme la réécriture de l'histoire précédente, et le roman entier comme une réécriture non pas de Marivaux, mais des Liaisons dangereuses, "une petite nouvelle du XVIII ${ }^{e}$ siècle revisitée [...], où [Camille/Claire] serai[t] à la fois Merteuil et Tourvel, manipulatrice et victime, celle qui meurt et celle qui tue" (CVC 188). De Marivaux à Choderlos de Laclos ou à Camille Laurens, le texte explore une même thématique qui lie l'art du mensonge à l'art du langage, et instaure un espace fictionnel qui transfère l'univers épistolaire et libertin du XvIII ${ }^{\mathrm{e}}$ siècle dans le monde de la réalité virtuelle, où l'on mystifie désormais par émojis interposés et profils trafiqués sur les réseaux sociaux.

La particularité du roman de Laurens tient toutefois à l'amputation systématique du discours: l'absence générale des destinataires et de leurs réponses, d'une histoire à l'autre, bâtit un modèle narratif de nature déficitaire. Et parce que le dialogue est tronqué, c'est alors bien le lectorat qui, par effet de ricochet, devient la cible première de ces propos mystificateurs, pris au piège de duperies adressées à des interlocuteurs absents et dont il est tenu d'endosser le rôle. Il y a donc bien ici une narration non fiable, mais dont la complexité tient surtout aux diverses échelles qu'elle implique : celle d'abord des personnages à la fois "manipulateurs" et "faillibles" par le doute pathologique qui plane régulièrement sur eux (Camille et Claire sont en séjour psychiatrique); celle d'autre part de ce que Booth nomme "l'auteur implicite", qui englobe les mécanismes narratifs généraux du roman (métalepses disposées en jeux de trompe-l'œil; suppression de fragments dialogiques) et suppose une manipulation discursive effectuée en amont, à un niveau extradiégétique.

\section{Esthétiques et politiques de la mystification}

Démultiplication de fausses pistes chez Laurens, pratique de la dissimulation chez NDiaye, ces voix fallacieuses du récit pourraient être lues dans une perspective facétieuse et ludique, rejoignant aux côtés d'auteurs tels que Jean Echenoz, Éric Chevillard ou Pierre Senges la joyeuse troupe des gais menteurs et des fictions espiègles qui font de la mystification littéraire, selon Maxime Decout, une pratique "jubilatoire" 27 . Or les textes de NDiaye et Laurens livrent aussi un discours plus sourdement critique qui questionne une société bâtie sur des faux-semblants, et invite à lire la duplicité comme un paradigme paradoxal de résistance.

La feintise en effet ne s'y réduit pas à la malice de l'instance narrative ou de quelque "auteur implicite": elle constitue la thématique principale des deux textes, contaminant l'espace romanesque et pour bonne part ses figurants. Chez NDiaye, les personnages mentent et dissimulent à tour de rôle : la mère feint de refaire sa vie et simule un bonheur conjugal douteux, le père feint d'ignorer sa cécité croissante, d'autres encore se travestissent ou se déguisent (Jenny s'obstine à "décolorer soigneusement ses cheveux" (AV 48)) tandis que les corps changent imperceptiblement de forme, grossissent ou s'amaigrissent. De manière plus éclatante encore chez 
Laurens, le mensonge s'étend à l'intégralité des normes sociales dans un univers diégétique hanté par la réalité virtuelle et le trucage des données personnelles (faux profils, fausses photos glanées sur Google, faux âge, faux roman etc.). En ce sens, le mensonge ne se limite donc plus uniquement à un acte individuel et intentionnel celui de l'instance narrative - mais se laisse également appréhender comme l'expression, le syndrome d'un phénomène collectif de nature presque pathologique. Moins proche de la définition d'Augustin que de celle, plus largement politique, qu'en propose Adorno dans ses Minima moralia, le mensonge est érigé en norme absolue et invisible qui règne sur une société devenue parfaitement inauthentique, "qui donne au faux les apparences du vrai, et au vrai celles du faux" 28 .

Chez Laurens, le caractère déceptif de la narration s'envisage donc aussi comme le prolongement logique, nécessaire, d'un ordre social mensonger, fondé sur les fauxsemblants, sur le diktat de l'image et sur des conventions sociales aliénantes dont diverses mystifications narratives se font le parangon critique. Car en exacerbant les procédés de duperie, la narration les démasque bien sûr du même coup, et Celle que vous croyez brosse sous la forme d'une diatribe vigoureuse le portrait amer d'une société misogyne dans laquelle les femmes sont soumises aux stéréotypes genrés du désir masculin et condamnées à devenir invisibles une fois passée leur "date de péremption" (CVC 143) :

tu le vois dans la rue tu le sens t'es vieille les regards me traversent et m'attaquent dégage casse-toi tu pues la mort tu sens le moisi vous avez vu Madonna les gens lui reprochent de "vouloir continuer à exister" c'est ça ce sont les mots exacts que j'ai lus dans le journal un vrai journal un journal sérieux "à cinquante-cinq ans Madonna est pathétique de vouloir continuer à exister" qu'est-ce qu'il faut alors il faut vouloir cesser d'exister il faut se retirer de soi-même comprendre qu'on n'a plus rien à faire là plus de place je n'ai plus de place je ne sais pas où me mettre tiroir cercueil aller dans la boîte il ne sert à rien d'être jeune sans être belle ni d'être belle sans être jeune les homme mûrissent les femmes vieillissent (CVC 13)

21 Sous la violence des mots et de phrases scandées sans ponctuation, le rapport à l'écriture se donne à lire depuis une nouvelle perspective: tromper, falsifier son identité, son âge, créer un profil de jeune fille inventé de toutes pièces, ne sont pas que des actes désespérés. Ils sont aussi des actes de résistance - une manière de prendre sa revanche sur ceux-là mêmes qui imposent un système normatif auquel tentent d'échapper les personnages.

La résistance s'organise également chez Marie NDiaye, mais prend des formes plus intimes et moins contestataires. Dans Autoportrait en vert, comme dans de nombreux autres romans de l'autrice, les conventions sociales pèsent comme une chape de plomb sur les personnages. Chacun feint, dissimule, se déguise pour maintenir à sa manière les faux-semblants de relations familiales distendues et délétères qui accaparent les monologues intérieurs inquiets de la narratrice ("Serait-il acceptable que je reçoive des confidences au sujet de mon père ?" ( $A V$ 41), "Est-ce qu'il est de mon ressort de veiller sur mes sœurs, de m'occuper d'elles, de leur montrer de l'affection ?" (AV 74), "Et n'estce pas déplacé de m'inviter à Noël, moi seule [...], comme si tout à coup [ma mère] n'avait plus de petits enfants ou ne les reconnaissait plus ?"29 (AV 77)). Dans un univers où les individus obéissent sans cesse à des normes obscures et oppressantes, cherchent à tout prix à s'intégrer à des communautés familiales ou sociales pourtant souvent aliénantes ${ }^{30}$, la dissimulation prend aussi des accents d'échappée libertaire : elle offre au sujet un espace de protection pour échapper aux regards indiscrets, un refuge où 
préserver son droit à l'opacité. Les dissimulations trompeuses de la narratrice n'en sont peut-être alors, paradoxalement, que plus authentiques, celle-ci fondant précisément dans le refus de se plier aux conventions littéraires du récit autobiographique la marque de son individualité.

S'opère là peut-être, chez Laurens comme chez NDiaye - et quoiqu'à des échelles distinctes - un renversement de ce que Sartre nomme la "mauvaise foi". À la différence du célèbre garçon de cafési ${ }^{31}$ les autrices-narratrices-personnages ne jouent pas à faire semblant d'être ce qu'elles prétendent être (ou ce qu'il conviendrait qu'elles soient), mais entreprennent au contraire de créer des failles entre un certain horizon d'attente (social comme littéraire) et la réalisation d'un projet d'écriture qui travaille à le transgresser. Tout s'y révèle alors acte de dissimulation: personnages menteurs à l'échelle diégétique, instances narratives brouillant la véracité du discours, ancrage générique enfin lui-même trompeur. Par son caractère déceptif, le texte fait ici acte de résistance. Une résistance esthétique d'abord, en contrevenant aux normes tacites de l'écriture de soi qui suppose une démarche introspective et un parti pris de sincérité deux présupposés que récusent les textes - ainsi qu'en instaurant plusieurs dispositifs de brouillage énonciatif (procédés de dispersion chez NDiaye, obstruction métaleptique chez Laurens, recours pour toutes deux à une narration non fiable placée sous le double sceau de la faillibilité et de la manipulation). Résistance politique enfin, parce que les textes procèdent à une prise de recul critique face à un espace normatif qu'ils reproduisent et transgressent à la fois par leurs voix fallacieuses. On pourrait en somme conclure que ces récits de soi sont d'autant plus efficaces qu'ils se présentent comme dissidents : par leurs accointances mystificatrices, ils posent la question de normes assujettissantes (ce pouvoir auquel ils nous lient aussi sourdement, nous lectrices et lecteurs) et récréent des zones d'échappement hors de tout carcan générique ou social où l'écriture du "moi" peut reconquérir une certaine liberté - celle notamment de célébrer le sujet dans toute la puissance déceptive de son opacité.

\section{NOTES DE FIN}

1. Ce pacte est décrit par Philippe Lejeune dans son ouvrage devenu canonique, Le pacte autobiographique, Paris, Éditions du Seuil, 1975.

2. Augustin, Du mensonge, in Euvres complètes de saint Augustin, vol. 12, éd. Raulx, Bar-le-Duc, Guérin, 1869, traduction par l'abbé Devoille, p. 195-217.

3. Pour une analyse détaillée et critique de la notion de mensonge au travers des siècles, et notamment de la définition augustinienne, je renvoie à Jochen Mecke, "Une critique du mensonge par-delà le bien et le mal", Cahiers d'Études Germaniques, , nº 67, "Quelques vérités à propos du mensonge ?" (vol. 1), Hélène Barrière, Karl Heinz Götze, Ingrid Haag (dirs), 2014, p. 91-110. 
4. Jochen Mecke, "Esthétique du mensonge", Cahiers d'Études Germaniques, nº 68, "Quelques vérités à propos du mensonge?" (vol. 2), Hélène Barrière, Susanne Böhmisch, Karl Heinz Götze, Ingrid Haag (dirs), 2015, p. 76-91.

5. Voir à ce sujet notamment Wayne C. Booth, "Distance et point de vue" [1966], in Gérard Genette, Tzvetan Todorov, Poétique du récit, Paris, Éditions du Seuil, 1977, p. 85-112, et Ansgar Nünning, "Unreliable, compared to what? Towards a cognitive theory of unreliable narration: prolegomena and hypotheses", in Walter Grünzweig, Andreas Solbach (dirs), Grenzüberschreitungen : Narratologie im Kontext, Tübingen, Gunter Narr, 1999, p. 53-73. Il convient toutefois de distinguer plusieurs types de narrations non fiables qui ne sont pas toutes nécessairement mensongères. Nous y reviendrons au cours de l'article.

6. Sur la variété des impostures littéraires (extra- comme intradiégétiques), voir Maxime Decout, Pouvoirs de l'imposture, Paris, Éditions de Minuit, 2018, <Paradoxe>.

7. Jean-Jacques Rousseau, Les confessions, Paris, Gallimard, 2009 [1782], <Folio classique>, p. 33.

8. Chloé Delaume, La règle du Je, Paris, Presses Universitaires de France, 2010, p. 67.

9. Marie NDiaye, Autoportrait en vert, Paris, Gallimard, 2009 [2005], <Folio>; dorénavant AV. Camille Laurens, Celle que vous croyez, Paris, Gallimard, 2019 [2016], <Folio> ; dorénavant CVC.

10. Dans Autofiction et autres mythomanies littéraires, (Auch, Tristram, 2004), Vincent Colonna rappelle certes que la veine fantastique constitue une composante riche du genre autofictionnel. Sa définition du terme de "fantastique" reste toutefois assez floue et se réfère en fait davantage aux modalités du merveilleux, avec ses monstres et ses enchantements, qu'à celles plus troubles du fantastique, tout en indéterminations et suppositions ténues - que l'on retrouve précisément dans Autoportrait en vert.

11. Au sujet des couleurs chez Marie NDiaye, je renvoie à l'article de Shirley Jordan, "Washes and hues: Reading for colour in Marie Ndiaye", French Studies, ${ }^{\circ} 71,2017$, p. 362-373.

12. La ressemblance avec Kafka tient ici au caractère paradoxal de la focalisation interne (pensons au personnage de Josef $\mathrm{K}$. dans Le Procès, qui affiche une étrangeté d'autant plus vive que ses pensées, bien que connues du lecteur, n'en restent pas moins parfaitement opaques). De Camus, NDiaye se rapproche davantage par la mise en scène d'une instance narrative autodiégétique et pourtant incernable (bien qu'il soit possible d'attribuer cet effet, dans L'étranger, à une focalisation interne qui mime une focalisation externe en en reprenant les principales caractéristiques).

13. Ceci est en outre rendu visible, dans la construction phrastique du texte, par un emploi récurrent de procédés de réticence et de suspension narrative, proches parfois de l'aposiopèse, qui fragmentent le discours et retardent, voire obstruent le processus heuristique qui le soustend.

14. Je me fonde ici sur les travaux d'Émile Benveniste, Problèmes de linguistique générale, I, Paris, Gallimard, 1966, p. 252.

15. On retrouve un passage très similaire : "il lui paraît invraisemblable qu'elle soit, elle, Jenny, dans la situation désolante où il semble bien qu'elle soit, elle Jenny, que la chance avait comblée de dons" (AV 52).

16. “Je dirai d'un narrateur qu'il est digne de confiance (reliable) quand il parle ou agit en accord avec les normes de l'œuvre (ce qui revient à dire : les normes implicites de l'auteur), et je le dirai indigne de confiance (unreliable) dans le cas contraire" Wayne C. Booth, "Distance et point de vue", art. cit., p. 105.

17. Je souligne.

18. Francis Langevin, "La posture exotique du narrateur-personnage. Inconfort et non-fiabilité dans quelques romans contemporains", in Frances Fortier, Andrée Mercier (dirs), La transmission narrative. Modalités du pacte romanesque contemporain, Montréal, Nota Bene, 2011, p. 207-233. Pour une étude panoramique des différentes théories et catégories de la narration non fiable, voir également Frank Wagner, "Quand le narrateur boit(e)... (Réflexions sur le narrateur non fiable 
et/ou indigne de confiance)", Arborescences, $\mathrm{n}^{\circ} 6$, "Polyphonies: voix et valeurs du discours littéraire", septembre 2016, p. 148-175.

19. Langevin subsume sous la notion d'exotisme trois types "d'altérité" qui expliquent la nonfiabilité du discours : altérité culturelle, altérité cognitive et altérité éthique. Voir ibid.p. 229.

20. Bien qu'improbable ici du fait du caractère strictement anecdotique de la mention, l'hypothèse n'est pas sans rappeler les inquiétants rajeunissements de figures maternelles dans d'autres romans de l'autrice, notamment Rosie Carpe.

21. Si la métalepse n'est pas nécessairement de nature fallacieuse, il n'est toutefois pas anodin que Gérard Genette la définisse précisément comme "une manipulation [...] de cette relation causale qui unit, dans un sens ou dans l'autre, l'auteur à son œuvre" (Gérard Genette, Métalepse. De la figure à la fiction, Paris, Éditions du Seuil, 2004, <Poétique>, p. 14, je souligne). Plus loin, il parlera d'"une illusion consistant à recevoir la fiction comme une réalité" (ibid., p. 51).

22. Camille Laurens s'en réclame d'ailleurs elle-même sans réticence. Voir à ce sujet son entretien $\mathrm{du}$ 12/06/2020 disponible sur France Culture: https://www.franceculture.fr/ emissions/les-masterclasses/camille-laurens-lautofiction-cest-insister-sur-ce-melange-defiction-a-loeuvre-y-compris-quand.

23. Philippe Lejeune, op. cit., p. 16. Lejeune mentionne, entre autres, l'exemple du Traître d'André Gorz.

24. Derrière lequel on est tenté de voir un rappel ironique du procès intenté justement par Camille Laurens à Marie Darrieussecq pour son roman Philippe. Les prises de position des deux intéressées sont rassemblées en deux textes publiés conjointement dans le volume de Claude Burgelin, Isabelle Grell, Roger-Yves Roche (dirs), Autofiction(s), Lyon, Presses Universitaires de Lyon, 2010, p. 495-506 et p. 507-523.

25. Voir, à titre d'exemples, Port intérieur d'Antoine Volodine (Paris, Éditions de Minuit, 1995), et Assommons les pauvres! de Shumona Sinha (Paris, Éditions de l'Olivier, 2011).

26. C'est d'ailleurs le titre choisi pour l'autofiction écrite par Claire - récit fictif enchâssé dans la narration du psychiatre.

27. "La jubilation [...] est une compagne fidèle des récits d'imposture et d'enquête. Motif inattendu, vous en conviendrez, mais motif essentiel. Preuve à l'appui : ces récits instaurent un rapport ludique, joueur, rusé, avec l'être, qui passe par l'écriture elle-même" (Maxime Decout, op. cit., p. 178).

28. Theodor W. Adorno, Minima moralia. Reflexionen aus dem beschädigten Leben, Frankfurt am Main, Suhrkamp, 1969, p. 77, je traduis. En allemand: "Es ist dahin gekommen, dass Lüge wie Wahrheit klingt, Wahrheit wie Lüge." Voir également : “Die Lüge [hat] längst ihre ehrliche Funktion verloren, über Reales zu täuschen [...]. Die Lüge, einmal liberales Mittel der Kommunikation, ist heute zu einer der Techniken der Unverschämtheit geworden, mit deren Hilfe jeder Einzelne die Kälte um sich verbreitet, in deren Schutz er gedeihen kann." (ibid., p. 28). 29. Je souligne pour les trois citations.

30. Les ambivalences du motif familial constituent un véritable leitmotiv de l'œuvre de Marie NDiaye, d'En famille (1990) à La sorcière (1996), Mon cœur à l'étroit (2007) ou Ladivine (2013).

31. Jean-Paul Sartre, L'être et le néant. Essai d'ontologie phénoménologique, Paris, Gallimard, 1943 $<$ Bibliothèque des idées>.

\section{RÉSUMÉS}

Le récit de soi ne compte pas généralement parmi les genres littéraires dans lesquels l'instance narrative a pour habitude de mentir, et l'idée de duplicité narrative semble de prime abord mal s'accommoder d'un pacte de lecture qui présuppose une exigence de sincérité. L'autofiction ellemême n'y déroge guère: la fiction, pour inventive qu'elle soit, n'équivaut pas au mensonge. 
Qu'en est-il en revanche lorsque la narration prend un malin plaisir à mener son lecteur en bateau et que la manipulation devient le parangon d'une esthétique introspective de nature déceptive, fondée sur la dissimulation ou sur le brouillage des pistes de lecture? L'article se propose de détailler ces pratiques à travers l'analyse d'Autoportrait en vert de Marie NDiaye (2005) et de Celle que vous croyez de Camille Laurens (2016). Il s'agira, d'une part, de revenir sur la singularité de ces textes par rapport aux pratiques autofictionnelles en insistant sur les procédés de tromperie narrative à l'œuvre chez les deux romancières (dispersions énonciatives teintées de fantastique chez l'une ; démultiplications métaleptiques et cryptage virtuel chez l'autre). D'autre part, il nous faudra sonder les enjeux de ces écritures : car si les mises en scène contemporaines de la mauvaise foi tendent à s'inscrire dans une pratique littéraire volontiers ludique, Marie NDiaye et Camille Laurens livrent un discours plus sourdement critique qui questionne les modalités de la représentation féminine dans une société normative, et invite à lire la duplicité comme paradigme de résistance face à des conventions (littéraires, sociales, genrées) que les textes s'appliquent à enfreindre par l'usage de voix fallacieuses.

\section{INDEX}

Mots-clés : récit de soi, mensonge, autofiction

\section{AUTEURS}

\section{ANNE-SOPHIE DONNARIEIX}

Universität Regensburg 